\title{
A New Multi-Method Combination Forecasting Model for ESDD Predicting
}

\author{
Haiyan SHUAI, Qingwu GONG \\ ${ }^{1}$ School of Electrical Engineering, Wuhan Technical College of Communications, \\ Wuhan University, Wuhan, China \\ ${ }^{2}$ School of Electrical Engineering, Wuhan University, Wuhan, China \\ Email:wdshy@126.com,whdxgqw@163.com
}

\begin{abstract}
Equal Salt Deposit Density (ESDD) is a main factor to classify contamination severity and draw pollution distribution map. The precise ESDD forecasting plays an important role in the safety, economy and reliability of power system. To cope with the problems existing in the ESDD predicting by multivariate linear regression (MLR), back propagation (BP) neural network and least squares support vector machines (LSSVM), a nonlinear combination forecasting model based on wavelet neural network (WNN) for ESDD is proposed. The model is a WNN with three layers, whose input layer has three neurons and output layer has one neuron, namely, regarding the ESDD forecasting results of MLR, BP and LSSVM as the inputs of the model and the observed value as the output. In the interest of better reflection of the influence of each single forecasting model on ESDD and increase of the accuracy of ESDD prediction, Morlet wavelet is used to construct WNN, error backpropagation algorithm is adopted to train the network and genetic algorithm is used to determine the initials of the parameters. Simulation results show that the accuracy of the proposed combination ESDD forecasting model is higher than that of any single model and that of traditional linear combination forecasting (LCF) model. The model provides a new feasible way to increase the accuracy of pollution distribution map of power network.
\end{abstract}

Keywords: equal salt deposit density, multivariate linear regression, BP neural network, least squares support vector machines, combination forecasting, wavelet neural network

\section{Introduction}

Exposed to dirty environment, surfaces of insulators will be polluted. After being wetted, the polluted layers will deduce insulators' insulation capability which often invites pollution flashover. According to the operation experiences of power sectors, pollution flashover is one of the main factors causing power accidents. In recent years $[1,2]$, there have happened several large area pollution flashovers in some locals or the whole country, which resulted in large area power blackout. Among all the causes resulting in pollution flashover, the lag and the inaccuracy of pollution distribution map of power network is a primary one. Equal Salt Deposit Density (ESDD) is the equivalent amount of $\mathrm{NaCl}$ that would yield the same conductivity at complete dilution [3], which is the dominant factor to classify contamination severity and draw pollution distribution map. At present, the methods of ESDD forecasting are mainly traditional multivariate linear regression (MLR) technique [4,5], back propagation (BP) neural network [6,7], and least squares support vector machines (LSSVM) [8,9]. Combination forecasting integrates the useful information of all single forecasting models and generally considers each forecasting result; hence it can more systemically and comprehensively reflect the changes of an object than a single model does. J. M. Bates and C. J. W. Granger proved the combination of two or more than two agonic single forecasting models can produce the result better than that of each single model, which showed combination forecasting method can increase prediction accuracy [10].

To improve ESDD forecasting accuracy, a nonlinear combination forecasting model based on wavelet neural network (WNN) for ESDD is proposed. The model is a WNN with three layers, whose inputs are the forecasting results of the three ESDD forecasting models mentioned above, i.e., MLR, BP and LSSVM, and outputs are the observed values of ESDD. For the sake of better reflection of the influence of each single forecasting model on ESDD and increase of the accuracy of ESDD prediction, Morlet wavelet is used to construct WNN and error backpropagation algorithm is adopted to train the network. Simulation results show that the accuracy of the proposed combination ESDD forecasting model is higher than that of any single model and also higher than that of 
traditional linear combined forecasting (LCF) model. The model provides a new doable way to boost the precision of pollution distribution map of power network.

\section{Brief Introductions of MLR, BP, LSSVM, and LCF}

\subsection{MLR Model}

If $y$ is a random variable predicted through $x_{1}, x_{2}, \cdots, x_{p}$ and there is a linear relationship between them, then a $p$-variable linear regression equation can be constructed as follow [11]:

$$
y=\beta_{0}+\beta_{1} x_{1}+\cdots+\beta_{p} x_{p}+\varepsilon
$$

where $p$ is the number of independent variables.

Considering the $n$ groups of data observed are as followings respectively:

$$
\left(x_{i 1}, x_{i 2}, \cdots, x_{i p} ; y_{i}\right) \quad i=1,2, \cdots, n
$$

Put $\left(x_{i 1}, x_{i 2}, \cdots, x_{i p} ; y_{i}\right) i=1,2, \cdots, n$ into (1), one can get

$$
\left\{\begin{array}{c}
y_{1}=\beta_{0}+\beta_{1} x_{11}+\beta_{2} x_{12}+\cdots+\beta_{p} x_{1 p}+\varepsilon_{1} \\
y_{2}=\beta_{0}+\beta_{1} x_{21}+\beta_{2} x_{22}+\cdots+\beta_{p} x_{2 p}+\varepsilon_{2} \\
\vdots \\
y_{n}=\beta_{0}+\beta_{1} x_{n 1}+\beta_{2} x_{n 2}+\cdots+\beta_{p} x_{n p}+\varepsilon_{n}
\end{array}\right.
$$

where $\beta_{0}, \beta_{1}, \cdots$, and $\beta_{p}$ are the $(p+1)$ parameters being estimated, called overall regression parameters; $\varepsilon_{1}, \varepsilon_{2}, \cdots$, and $\varepsilon_{n}$ are random errors independent with each other, and $\varepsilon_{i} \sim N\left(0, \sigma^{2}\right)$.The task of multivariable linear regression is to solve the following three problems according to (2).

1) Determine $\hat{\beta}_{0}, \hat{\beta}_{1}, \cdots$, and $\hat{\beta}_{p}$ and the regression equation.

2) Carry out significant test to the regression equation. If the equation is representative, it is significant and can be used; otherwise, it can not be adopted.

3) Use the regression equation to predict or control the dependent variable under significant condition.

\subsection{BP Model}

BP $[6,7]$ is a kind of feed forward neural network. Supposing a BP network has $m$ layers, and each layer has several neurons. The neuron $j$ of layer $k$ has such an input-output relationship being described as:

$$
\begin{aligned}
& x_{j}^{k}=f_{j}^{(k)}\left[\sum_{i=1}^{n_{k-1}} \omega_{i j}^{(k)} x_{i}^{(k-1)}-b_{j}^{(k)}\right] \\
& \left(j=1,2, \cdots, n_{k} ; k=1,2, \cdots, m\right)
\end{aligned}
$$

where $\omega_{i j}^{(k)}$ is the connection weight between neuron $i$ of layer $(k-1)$ and neuron $j$ of layer $k ; b_{j}^{(k)}$ is the threshold of neuron $j$; the activation function $f_{j}^{(k)}$ is a sigmoidal function, i.e., $f_{j}^{(k)}(x)=\frac{1}{(1+\exp (-x))}$; and $n_{k}$ is the number of neurons of layer $k$.

\subsection{LSSVM Model}

LSSVM $[12,13]$ is described as following:

Considering a given training set of $m$ data points $\left\{x_{k}, y_{k}\right\}_{k=1}^{m}$ where $x_{k} \in R^{n}$ is the $k t h$ input vector and $y_{k} \in R$ is the kth output. In the feature space SVM models take the form:

$$
y(x)=\boldsymbol{\omega}^{T} \varphi(x)+b
$$

where the nonlinear mapping $\varphi(\cdot)$ maps the input data into a higher dimension feature space. The term $\mathrm{b}$ is a bias term. In least squares support vector machines for function estimation the following optimization problem is formulated:

$$
\begin{aligned}
& \min _{\omega, b, e} \mathbf{J}(\boldsymbol{\omega}, e)=\frac{1}{2} \boldsymbol{\omega}^{T} \boldsymbol{\omega}+\frac{1}{2} \gamma \sum_{i=1}^{m} e_{k}^{2} \\
& \text { s.t. } \quad y_{k}=\boldsymbol{\omega}^{T} \varphi\left(\mathbf{x}_{k}\right)+b+e_{k} \quad k=1, \ldots, m
\end{aligned}
$$

where $\gamma$ is a positive real constant and should be considered as a tuning parameter in the algorithm.

The Lagrangian is given by

$$
\mathbf{L}(\boldsymbol{\omega}, b, e, \alpha)=\mathbf{J}(\boldsymbol{\omega}, e)-\sum_{k=1}^{m} \alpha_{k}\left\{\omega^{T} \varphi\left(\mathbf{x}_{k}\right)+b+e_{k}-y_{k}\right\}
$$

where $\alpha_{k}$ are Lagrange multipliers, which can be either positive or negative due to the equality constraints as from the Kuhn-Tucker conditions.

The conditions for optimality are

$$
\left\{\begin{array}{l}
\frac{\partial \mathbf{L}}{\partial b}=0 \rightarrow \sum_{k=1}^{m} \alpha_{k}=0 \\
\frac{\partial \mathbf{L}}{\partial e_{k}}=0 \rightarrow \alpha_{k}=\gamma e_{k} \quad k=1,2,3 \ldots, m \\
\frac{\partial \mathbf{L}}{\partial \alpha_{k}}=0 \rightarrow \boldsymbol{\omega}^{T} \varphi\left(\mathbf{x}_{k}\right)+b+e_{k}-y_{k}=0 \quad k=1,2,3, \ldots m \\
\frac{\partial \mathbf{L}}{\partial \boldsymbol{\omega}}=0 \rightarrow \omega=\sum_{i=1}^{m} \alpha_{k} \varphi\left(\mathbf{x}_{k}\right)
\end{array}\right.
$$

After elimination of $\omega$ and $e$, one can get

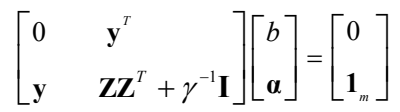

where

$$
\begin{aligned}
& \mathbf{y}=\left[y_{1}, y_{2}, \ldots, y_{m}\right], \mathbf{1}_{m}=[1,1, \ldots, 1], \boldsymbol{\alpha}=\left[\alpha_{1}, \alpha_{2}, \ldots, \alpha_{m}\right], \\
& \mathbf{z}=\left[\varphi\left(\mathbf{x}_{1}\right), \varphi\left(\mathbf{x}_{2}\right), \ldots, \varphi\left(\mathbf{x}_{m}\right)\right]
\end{aligned}
$$


From application of the Mercer condition one can obtain $\boldsymbol{\Omega}=K\left(\mathbf{x}_{i}, \mathbf{x}_{j}\right)=\varphi\left(\mathbf{x}_{i}\right)^{T} \varphi\left(\mathbf{x}_{j}\right), i, j=1,2, \ldots, m$. This finally results into the following LS-SVM model for function estimation

$$
y=\sum_{k=1}^{m} \alpha_{k} K\left(\mathbf{x}, \mathbf{x}_{k}\right)+b
$$

\subsection{LCF Model}

Linear combination forecasting model [14] takes the form:

$$
\left\{\begin{array}{l}
g_{t}=k_{1} g_{1 t}+k_{2} g_{2 t}+k_{3} g_{3 t} \\
k_{1}+k_{2}+k_{3}=1 \quad\left(k_{i} \geq 0, i=1,2,3\right)
\end{array}\right.
$$

where $g_{t}$ is the value of the linear combination forecasting model; $g_{1 t}, g_{2 t}$ and $g_{3 t}$ are the forecasting values of the single forecasting models respectively, namely, MLR, BP and LSSVM; and $k_{1}, k_{2}$ and $k_{3}$ are the weighted coefficients of the three single models respectively. To the linear combination forecasting method, its main task is to adopt optimization measures to obtain the optimal weighted coefficients which can make the error sum of squares of the combined forecasting be least.

\section{WNN Combination Forecasting Model for ESDD Predicting}

Supposing $z_{1 i}, z_{2 i}$ and $z_{3 i}$ are the ith ESDD predicting values of MLR, BP and LSSVM respectively, $h_{i}$ is the ith measured value. One can use $z_{1 i}, z_{2 i}$ and $z_{3 i}$ to construct a nonlinear combination function [15]

$$
\hat{h}_{i}=\varphi_{i}(Z)=\varphi_{i}\left(z_{1 i}, z_{2 i}, z_{3 i}\right)
$$

where, $\varphi$ is the nonlinear function and $\hat{h}_{i}$ is the combined forecasting value. In this paper, WNN is used to design the nonlinear function $\varphi$.

It is known the nature of wavelet transform [16] is an integral transform between different parameters.

$$
w_{a b}(a, b)=\int_{-\infty}^{+\infty} f(x) g(a, b, x) d t
$$

where, $g(a, b, x)=\frac{1}{\sqrt{|a|}} g\left(\frac{x-b}{a}\right)$ is called wavelet basis and $g(x)$ called mother wavelet. $a$ and $b$ are termed as scaling factor and translation factor of $g(a, b, x)$ respectively. To $f(x)$, the resolution of its local structure can be realized through adjusting $a 、 b$, namely, gearing the scale and position of wavelet basis window.

WNN [17] is a model which, based upon wavelet analysis, possesses neural network thread. In other words, WNN adopts nonlinear wavelet basis to replace common-used Sigmoid function in traditional neural network. Through linear superposition of nonlinear wavelet basis selected, the combination of the ESDD data of all single forecasting models is realized. The nonlinear combination function in (11) can be fitted as follow, by adopting wavelet basis $g(a, b, x)$ :

$$
\hat{h}_{i}=\varphi_{i}(Z)=\sum_{k=1}^{m} \omega_{k} g\left(\frac{\sum_{j=1}^{L} v_{j k} z_{j i}-b_{k}}{a_{k}}\right)
$$

where $\varphi_{i}(Z)$ is the ESDD value of nonlinear combination forecasting corresponding to the measured one $h_{i}$; $z_{j i}$ expresses the ith prediction value of the $j t h$ model, $L=3 ; Z=\left(z_{1 i}, z_{2 i}, z_{3 i}\right) ; \omega_{k} 、 v_{j k} 、 b_{k} 、 a_{k}$ are the weighted coefficient between output terminal and the $k t h$ hidden layer node, the weighted between the $j t h$ input and the $k$ th hidden layer node, the translation factor and scaling factor of the kth wavelet basis respectively; the number of wavelet basis $m$ is 7, according to the empirical formula $2 n+1$. Considering Morlet wavelet possessing relatively good localization and smoothness, it will be selected in (13).

$$
g(x)=\cos (1.75 x) \exp \left(-\frac{x^{2}}{2}\right)
$$

Figure 1 shows the structure of WNN. There are 3 input nodes- $z_{1 i}, z_{2 i}, z_{3 i}$ and one output node- $\varphi_{i}(Z)$.

The objective of using WNN to carry out regression analysis is to determine the network parameters- $\omega_{k}$ 、 $v_{j k} 、 b_{k}$ and $a_{k}$ by which $\varphi_{i}(Z)$ can be fitted optimally with $h_{i} . \omega_{k} 、 v_{j k} 、 b_{k}$ and $a_{k}$ can be optimized via Minimum Mean Square Error (MMSE) energy function as follow.

$$
\left.E=\frac{1}{2} \sum_{i=1}^{I}\left[\sum_{k=1}^{m} \omega_{k} g\left(\frac{\sum_{j=1}^{L} v_{j k} z_{j i}-b_{k}}{a_{k}}\right)-h_{i}\right)\right]^{2}
$$

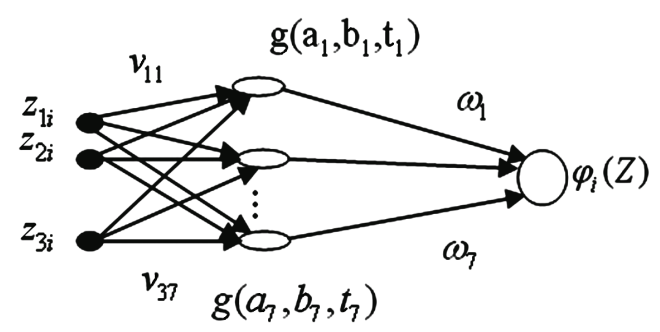

Figure1. The structure of WNN 
where, $I$ is the number of training samples and $h_{i}$ is the ith observed value.

To gain the optimal $\omega_{k} 、 v_{j k} 、 b_{k}$ and $a_{k}$ is to minimize (15). In this article, gradient descent algorithm is used as WNN learning principle. The details are as followings:

1) Set the objective error function value;

2) Initialize $\omega_{k} 、 v_{j k}, b_{k}$ and $a_{k}$ between $[-1,1]$ by using Genetic Algorithm [18];

3) Select randomly a training mode, and input the learning samples $z_{j i}$ and corresponding output $h_{i}$;

4) Work out the predicting value of the network $\hat{h}_{i}$, the followings are the details:

a) Calculate the gradient of each parameter;

Let $z^{*}=\sum_{j=1}^{L} v_{j k} z_{j i}, \quad S=\frac{z^{*}-b_{k}}{a_{k}}$, then the gradients of (15) are respectively:

$$
\begin{aligned}
& \frac{\partial E}{\partial \omega_{k}}=-\sum_{i=1}^{I}\left[\varphi_{i}(Z)-h_{i}\right] g\left[\frac{z^{*}-b_{k}}{a_{k}}\right] \\
\frac{\partial E}{\partial v_{j k}}= & -\sum_{i=1}^{I}\left[\varphi_{i}(Z)-h_{i}\right] \omega_{k}\left[-\cos (1.75 S) \exp \left(-\frac{S^{2}}{2}\right) \frac{S}{a_{k}}\right. \\
& \left.-1.75 \sin (1.75 S) \exp \left(-\frac{S^{2}}{2}\right) \frac{1}{a_{k}}\right] z_{j i} \\
\frac{\partial E}{\partial b_{j k}}= & -\sum_{i=1}^{I}\left[\varphi_{i}(Z)-h_{i}\right] \omega_{k}\left[\cos (1.75 S) \exp \left(-\frac{S^{2}}{2}\right) \frac{S}{a_{k}}\right. \\
& \left.+1.75 \sin (1.75 S) \exp \left(-\frac{S^{2}}{2}\right) \frac{1}{a_{k}}\right] \\
\frac{\partial E}{\partial a_{k}}= & -\sum_{i=1}^{I}\left[\varphi_{i}(Z)-h_{i}\right] \omega_{k}\left[\cos (1.75 S) \exp \left(-\frac{S^{2}}{2}\right) \frac{S^{2}}{a_{k}}\right. \\
& \left.+1.75 \sin (1.75 S) \exp \left(-\frac{S^{2}}{2}\right) \frac{S}{a_{k}}\right]
\end{aligned}
$$

b) Introduce momentum factor $\alpha$ to amend each parameter;

$$
\begin{aligned}
& \omega_{k}(t+1)=\omega_{k}(t)-\eta \frac{\partial E}{\partial \omega_{k}}+\alpha \Delta \omega_{k}(t) \\
& v_{j k}(t+1)=v_{j k}(t)-\eta \frac{\partial E}{\partial v_{j k}}+\alpha \Delta v_{j k}(t) \\
& b_{k}(t+1)=b_{k}(t)-\eta \frac{\partial E}{\partial b_{k}}+\alpha \Delta b_{k}(t) \\
& a_{k}(t+1)=a_{k}(t)-\eta \frac{\partial E}{\partial a_{k}}+\alpha \Delta a_{k}(t)
\end{aligned}
$$

where, $\eta$ is the learning ratio and $\alpha$ is the momentum factor.

c) Compute current output of WNN: put current parameters into (13) to get current output of the network;

d) Numerate error function value. When the error is less than the set one, the learning process is terminated; otherwise, turns to step (3).

\section{Appraisal of Forecasting}

In order to evaluate the prediction effects of the combination model comprehensively, according to the practice and principle, the paper uses relative error $\delta$ and average relative error $\bar{\delta}$ to evaluate the accuracy of the prediction.

$$
\begin{gathered}
\delta=\frac{\left|y_{i}-\hat{y}_{i}\right|}{y_{i}} \times 100 \% \\
\bar{\delta}=\frac{1}{I} \sum_{i=1}^{I} \frac{\left|y_{i}-\hat{y}_{i}\right|}{\mathrm{y}_{\mathrm{i}}} \times 100 \%
\end{gathered}
$$

where $y_{i}$ are the observed values and $\hat{y}_{i}$ are the predicted ones. These values reflect comprehensively the prediction results. The smaller $\delta$ and $\bar{\delta}$ are, the better the generalization of the model and the corresponding parameters are.

\section{Experimental Results}

On the basis of the model principles and modeling steps in the text, Matlab 7.0 is adopted to write the ESDD prediction programs based on WNN. References [4-7,9] show there is a close relationship between insulators ESDD and meteorological factors. So, for each single model, 120 samples of the historical meteorological data and ESDD data provided by "Optical Sensor System for the ESDD Monitoring of Transmission Equipment" (developed by Wuhan High Voltage Research Institute and Wuhan Kangpu Changqing Software Company) installed on the ESDD monitoring spot of Qingshan District, Wuhan ,from April to June in 2006, are regarded as training set and forecasting set, where 90 samples belong to training set, and the rest 30 samples to test set. Table 1 shows the comparisons between WNN combination forecasting model and LCF, MLR, BP and LSSVM.

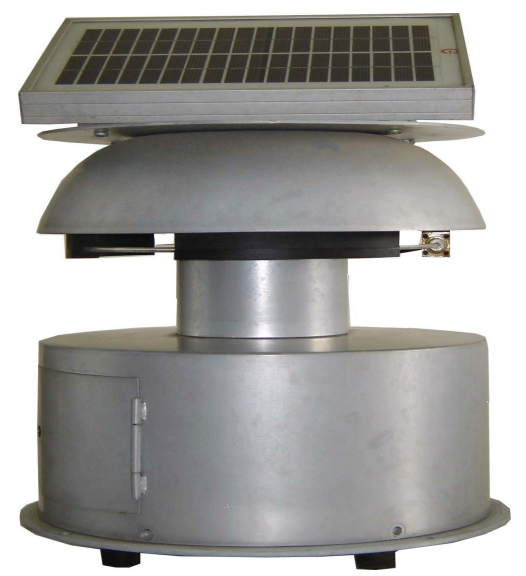

Figure 2. Optical sensor system for the ESDD monitoring of transmission equipment 
Table 1. Comparison of forecasting results of ten ESDD

\begin{tabular}{|c|c|c|c|c|c|c|c|c|c|c|c|}
\hline \multirow{3}{*}{ Times } & \multicolumn{7}{|c|}{ Combination forecasting } & \multicolumn{4}{|c|}{ Single forecasting } \\
\hline & \multicolumn{3}{|c|}{ WNN } & \multicolumn{2}{|l|}{ LCF } & \multicolumn{2}{|l|}{ MLR } & \multicolumn{2}{|l|}{$\mathrm{BP}$} & \multicolumn{2}{|c|}{ LSSVM } \\
\hline & $\begin{array}{l}\text { Actual } \\
\left(\mathrm{mg} / \mathrm{cm}^{2}\right)\end{array}$ & $\begin{array}{c}\text { Forecasting } \\
\left(\mathrm{mg} / \mathrm{cm}^{2}\right)\end{array}$ & $\delta(\%)$ & $\begin{array}{c}\text { Forecasting } \\
\left(\mathrm{mg} / \mathrm{cm}^{2}\right)\end{array}$ & $\delta(\%)$ & $\begin{array}{c}\text { Forecasting } \\
\left(\mathrm{mg} / \mathrm{cm}^{2}\right)\end{array}$ & $\delta(\%)$ & $\begin{array}{c}\text { Forecasting } \\
\left(\mathrm{mg} / \mathrm{cm}^{2}\right)\end{array}$ & $\delta(\%)$ & $\begin{array}{c}\text { Forecasting } \\
\left(\mathrm{mg} / \mathrm{cm}^{2}\right)\end{array}$ & $\delta(\%)$ \\
\hline 2006.4 .8 & 0.0268 & 0.0278 & 3.73 & 0.0252 & 5.97 & 0.0286 & 6.72 & 0.0249 & 7.09 & 0.0284 & 5.97 \\
\hline 2006.4.16 & 0.0263 & 0.0271 & 3.04 & 0.0276 & 4.94 & 0.0291 & 10.65 & 0.0247 & 6.08 & 0.0286 & 8.75 \\
\hline 2006.4 .21 & 0.0390 & 0.0381 & 2.31 & 0.0375 & 3.85 & 0.0412 & 5.64 & 0.0421 & 7.95 & 0.0363 & 6.92 \\
\hline 2006.5 .3 & 0.0353 & 0.0362 & 2.55 & 0.0367 & 3.97 & 0.0331 & 6.23 & 0.0334 & 5.38 & 0.0342 & 3.12 \\
\hline 2006.5 .8 & 0.0232 & 0.0244 & 5.17 & 0.0244 & 3.61 & 0.0248 & 6.45 & 0.0251 & 8.19 & 0.0247 & 6.47 \\
\hline 2006.5 .13 & 0.0264 & 0.0255 & 3.41 & 0.0251 & 4.92 & 0.0239 & 9.47 & 0.0292 & 10.61 & 0.0244 & 7.58 \\
\hline 2006.5.21 & 0.0487 & 0.0497 & 2.05 & 0.0517 & 6.16 & 0.0534 & 9.65 & 0.0527 & 8.21 & 0.0460 & 5.54 \\
\hline 2006.6 .1 & 0.0432 & 0.0414 & 4.17 & 0.0421 & 2.55 & 0.0463 & 7.17 & 0.0410 & 5.09 & 0.0455 & 5.32 \\
\hline 2006.6 .9 & 0.0459 & 0.0442 & 3.70 & 0.0481 & 4.79 & 0.0491 & 6.97 & 0.0427 & 6.97 & 0.0482 & 5.01 \\
\hline 2006.6.18 & 0.0467 & 0.0484 & 3.64 & 0.0491 & 5.14 & 0.0411 & 11.99 & 0.0488 & 4.50 & 0.0451 & 3.43 \\
\hline $\bar{\delta}(\%)$ & & & 3.377 & & 4.590 & & 8.094 & & 7.007 & & 5.811 \\
\hline
\end{tabular}

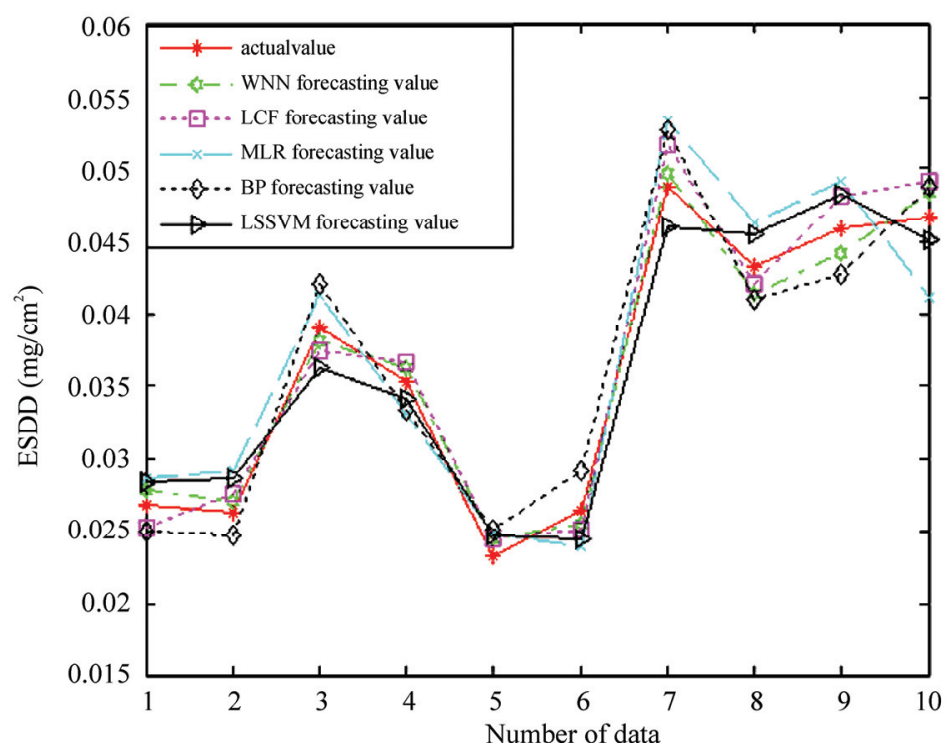

Figure 3. Comparison between the predicted and the measured

From Table 1, it can be seen that the average relative error of MLR is $8.094 \%$ which is the biggest one among those of the five forecasting models, and that of $\mathrm{WNN}$ is $3.377 \%$, the least one, and each relative error of $\mathrm{WNN}$ is less than $6 \%$. The average relative error of LCF is $4.590 \%$ which is only bigger than that of WNN. Besides, the maximum relative error of LCF, MLR, BP and LSSVM are $6.16 \%, 11.99 \%, 10.61 \%$ and $7.58 \%$ respectively; while that of $\mathrm{WNN}$ is only $5.17 \%$. This indicates that the prediction accuracy of combination model is higher than that of each single model, and compared with linear combination model, WNN combination model presented in the paper is more stable and practical, and can improve efficiently forecasting precision. Using the ESDD predicted by WNN combination forecasting model to divide pollution area can effectively increase the accuracy of pollution distribution map.

\section{Conclusions}

ESDD is a main factor to classify contamination severity and draw pollution areas map of power network, hence its accuracy directly influences the precision of pollution 
distribution map, and further affects the insulation capability of power system.

The WNN combination forecasting model combined with multivariable linear regression technique, BP neural network and least squares support vector machines avoids the limitations of linear combination model and single forecasting model, so it can boost the forecasting accuracy, especially deduce maximum relative error, in other words, it can decline predicting risk. The simulation results in Table 1 show in the five models, the prediction accuracy of WNN combination model is the highest one, namely, the values of $\mathrm{WNN}$ are closest to the observed ones. The ESDD values produced by WNN combination forecasting model can better meet the request of drawing pollution distribution map of power network. The model proposed by the paper is an effective and doable way for ESDD forecasting and provides a new thinking for the computerization of drawing pollution distribution map.

\section{REFERENCES}

[1] Y. Hu, "The analysis of ' 2.22 pollution flashover of network' and measure for anti-pollution flashover," High Voltage Engineering, Vol. 27, pp. 30-32, April 2001.

[2] Y. Liu and J. K. Wang, "Analysis of large area pollution flashover occurred in Shaanxi power network on Dec.18, 2000 and preventative measures for similar accidents," Power System Technology, Vol. 26, pp. 82-85, June 2002.

[3] Pollution Classification and External Insulation Selection for Electrical Power System, State Grid Standard Q/ GDW152-2006, December 2006.

[4] A. S. Almad, H. Ahmad, M. A. Salam, and Saad Ahmad, "Regression technique for prediction of salt contamination severity on high voltage insulators," in Annual Report Conference on Electrical Insulation and Dielectric Phenomena, pp. 218-221, 2000.

[5] A. S. Almad, H. Ahmad, M. A. Salam, T. Tamsir, Z. Buntat, and M. W. Mustafa, "Prediction of salt contamination on high voltage insulators in rainy season using regression technique," Proceedings of TENCON, Vol. 3, pp. 184-189, 2000.

[6] M. A. Salam, S. M. Ai-Alawi, and A. A Maqrashi, "Prediction of equivalent deposit density of contaminated glass plates using artificial neural networks," Journal of Electrostatics, Vol. 66, pp. 526-530, May 2008.
[7] A. S. Almad, P. S. Ghosh, H. Ahmad, and S. A.K. Aljunid, "Assessment of ESDD on high-voltage insulators using artificial neural network," Electric Power System Research, Vol. 72, pp. 131-136, December 2004.

[8] S. B. Jiao, D. Liu, G. Zheng, and Q. Zhang, "Forecasting the ESDD of insulator based on least squares support vector machine," Proceedings of the CSEE, Vol. 26, pp. 149-153, January 2006.

[9] F. H. Shu and W. X. Zhang, "A prediction model for insulator's ESDD based on least square support vector machine," High Voltage Apparatus, Vol. 44, pp. 420-423, October 2008.

[10] J. M. Bate and C. W. J. Granger, "The combination of forecastings," Operational Research Quarterly, Vol. 20, pp. 451-468, 1969.

[11] Y. Liu. "Mathematical model of multivariable linear regression," Journal of Shenyang Institute of Engineering, Vol. 1, pp. 128-129, Junuary 2005.

[12] J. A. K. Suykens and J. Vandewalle. "Least squares support vector machine classifiers," Neural Process Letters, Vol. 9, pp. 293-300, 1999.

[13] J. A. K. Suykens, L. Lukas, P. Van Dooren, B. Demoor, and J. Vandewalle, "Least squares support vector machine classifiers: A large scale algorithm,” ECCTD'99 European Conference on Circuit Theory and Design, pp. 293-300, 1999.

[14] Y. K. Ma and X. W. Tang, "Research on the problem of optimizing linear combination forecasting model," System Engineering - Theory \& Practice, Vol. 9, pp. 110123, September 1998.

[15] X. H. Wen and M. J. Niu, "A new nonlinear combined forecasting method on the basis of neural networks," System Engineering-Theory \& Practice, Vol. 12, pp. 66-72, December 1994.

[16] S. X. Li, "Wavelet transform and its applications," Higher Education Press, Beijing, pp. 11-15, 1997.

[17] Q. X. Cuo and L. Liu, "Driving force prediction for inclusion complexation of 2-cyclodextrim with benzene derivatives by a wavelet neural network," Chemical Physics Letters, Vol. 290, pp. 514-518, 1998.

[18] H. F. Liang, G. Y. Tu, and H. W. Tang, "Application of genetic algorithm neural network for short term load forecasting of power system," Power System Technology, Vol. 25, pp. 49-53, January 2001. 